\title{
Complicated Isolated Liver Abscess Caused by Viridans Group Streptococci Leading to Right Hepatectomy
}

\author{
Muhammad F. Ahmed ${ }^{1}$, Zainab Abbasi ${ }^{2}$, Sajan Das ${ }^{3}$, Alok Aggarwal ${ }^{4}$, Sonu Sahni 1, 5, 6 \\ 1. Internal Medicine, Brookdale University Hospital Medical Center, Brooklyn, USA 2. Internal Medicine, Liaquat \\ University of Medical and Health Sciences, Jamshoro, PAK 3. Internal Medicine, Kingsbrook Jewish Medical Center, \\ Brooklyn, USA 4. Surgery, Brookdale University Hospital Medical Center, Brooklyn, USA 5. Research Medicine, New \\ York Institute of Technology College of Osteopathic Medicine, New York, USA 6. Primary Care, Touro College of \\ Osteopathic Medicine, New York, USA
}

Corresponding author: Sonu Sahni, sonu.sahni@touro.edu

\begin{abstract}
Pyogenic liver abscesses (PLAs) secondary to bacterial etiologies are most often seen in developing countries and are less common in North America. The predominant etiology is infection occurring in the setting of direct extension of hepatobiliary or intestinal infection. The most common pathogen isolated from a PLA in the United States is Escherichia coli, whereas Streptococcus viridans is a rare entity in the developed world. Herein we report a rare case of a complicated isolated PLA in a patient without any known comorbidities which lead to hepatectomy. The patient was born and raised in the United States with no recent travel history. The patient was found to have $10 \mathrm{~cm}$ isolated multicystic mass on imaging confirmed later as vancomycin-resistant Streptococcus viridans PLA. The patient was treated with multiple intravenous antibiotics and underwent multiple ultrasound-guided percutaneous abscess drainages by interventional radiology, but all unsuccessful. The patient underwent right posterior liver lobectomy, thereafter making a quick recovery and was discharged. Our case underlines the significance of considering liver abscess as a differential even in previously healthy individuals with no known prior comorbid conditions, as prompt recognition is imperative in preventing morbidity and mortality.
\end{abstract}

Received 06/29/2020

Review began 07/04/2020 Review ended 07/06/2020 Published 07/12/2020

(c) Copyright 2020

Ahmed et al. This is an open access article distributed under the terms of the Creative Commons Attribution License CC-BY 4.0., which permits unrestricted use, distribution, and reproduction in any medium, provided the original author and source are credited.
Categories: Gastroenterology, General Surgery, Infectious Disease

Keywords: pyogenic liver abscess, streptococcus viridans, hepatectomy, ultrasound-guided drianage

\section{Introduction}

Viridans group streptococci (VGS) are a large group of commensal bacteria, most commonly a part of human oral flora and live in close association with the gingiva and the teeth. These catalase-negative gram-positive organisms are further divided into five groups: Streptococcus mitis group, Streptococcus anginosus group, Streptococcus bovis group, Streptococcus mutans group, and Streptococcus salivarius group [1]. Each of these groups potentially leads to a distinct disease presentation varying from bacteremia, abscess formation to endocarditis if introduced into the bloodstream. Pyogenic liver abscess (PLA) is a liver abscess caused by bacterial infection, most commonly by intestinal flora. It is most often seen in seen in developing countries and is less commonly seen in North America with an incidence of approximately 2.3 cases per 100,000 [2]. VGS are now being increasingly recognized as an opportunistic disease-causing organism in an immunocompromised host but rarely presents in PLA [3]. The pathomechanism of PLA is that infection which occurs in the setting of direct extension hepatobiliary or intestinal infection and hematogenous spread [4]. Herein we report a unique case of an otherwise healthy individual with no known comorbid conditions that presented with a large pyogenic hepatic abscess caused by VGS that remained resistant to conventional medical and interventional management strategies and ultimately right hepatectomy was performed as a curative treatment.

\section{Case Presentation}

A 52-year-old African American male with no significant past medical history presented to the emergency department with a chief complaint of sudden onset of right-sided abdominal pain that continued to worsen for a few days prior to presentation. On day of presentation, the patient stated that the pain had become excruciating rated 10/10 in severity which he described as dull in nature, non-radiating with no aggravating or relieving factors. He also complained of associated right-sided chest and flank pain. He reported subjective fevers but denied any chills, nausea, vomiting, diarrhea, measured weight loss, or any recent travels outside of the United States. Social history was insignificant.

On presentation, the patient was noted to be tachycardic with a fever of $102.5^{\circ} \mathrm{F}\left(39.2^{\circ} \mathrm{C}\right)$, respiratory rate of 20 breaths per minute, saturating at $99 \%$ on room air, and blood pressure of $115 / 65 \mathrm{mmHg}$. He was noted to be alert, awake, oriented, and was in mild distress. Ocular examination showed icterus. Throat and neck examination were completely benign, with no oral lesions or lymphadenopathy. Chest examination revealed 


\section{Cureus}

normal heart sounds, and lung sounds were also normal and vesicular. His abdomen was soft and distended with tenderness noted in all quadrants. Murphy's sign was negative. Bowel sounds were normal in all quadrants. No masses were palpated otherwise.

Laboratory studies demonstrated hemoglobin of $12.2 \mathrm{~g} / \mathrm{dL}$, white blood cell (WBC) count of $12.10 \times 10^{9} / \mathrm{L}$ with $77.1 \%$ neutrophils, and platelets of $137 \times 10^{9} / \mathrm{L}$. The metabolic panel revealed normal blood urea nitrogen and creatinine levels. However, liver enzymes were elevated with an alanine aminotransferase (ALT) level of $237 \mathrm{U} / \mathrm{L}$, aspartate aminotransferase (AST) $236 \mathrm{U} / \mathrm{L}$, alkaline phosphatase of within normal range, total bilirubin $1.8 \mathrm{mg} / \mathrm{dL}$, and lactate was normal at $1.4 \mathrm{mmol} / \mathrm{L}$. The patient was also tested for hepatitis B and C markers, which came out to be negative. His initial laboratory findings are summarized in Table 1.

\begin{tabular}{|c|c|c|}
\hline & Results & Reference Range \\
\hline White Blood Cell Count & 12.10 & $4.10-10.10 \times 10^{9} / L$ \\
\hline Red Blood Cell Count & 5.45 & $4.33-5.43 \times 10^{12} / \mathrm{L}$ \\
\hline Hemoglobin & 12.2 & $13.4-15.4 \mathrm{~g} / \mathrm{dL}$ \\
\hline Hematocrit & 37.9 & $40.0 \%-47.0 \%$ \\
\hline Mean Corpuscular Volume & 69.4 & 80.8-94.1 fL \\
\hline Platelet Count & 137 (L) & $153-328 \times 10^{9} / \mathrm{L}$ \\
\hline Neutrophils Absolute & $9.40(\mathrm{H})$ & $1.40-6.80 \times 10^{9} / \mathrm{L}$ \\
\hline Lymphocytes Absolute & 0.40 (L) & $1.10-2.90 \times 10^{9} / \mathrm{L}$ \\
\hline Monocytes Absolute & $2.30(\mathrm{H})$ & $0.20-1.00 \times 10^{9} / \mathrm{L}$ \\
\hline Eosinophils Absolute & 0 & $0.00-0.40 \times 10^{9} / \mathrm{L}$ \\
\hline Basophils Absolute & 0.10 & $0.00-0.10 \times 10^{9} / \mathrm{L}$ \\
\hline Prothrombin Time & $21.8(H)$ & 9.2-12.8 seconds \\
\hline International Normalized Ratio & $1.91(\mathrm{H})$ & $0.70-1.20$ \\
\hline Partial Thromboplastin Time & 30.7 & 23.5-35.5 seconds \\
\hline Glucose & $132(\mathrm{H})$ & $70-99 \mathrm{mg} / \mathrm{dL}$ \\
\hline Blood Urea Nitrogen & 15 & $9.0-20.0 \mathrm{mg} / \mathrm{dL}$ \\
\hline Creatinine & 0.89 & $0.66-1.25 \mathrm{mg} / \mathrm{dL}$ \\
\hline Sodium & 140 & 133-145 mEq/L \\
\hline Potassium & 4.5 & 3.5-5.1 mEq/L \\
\hline Chloride & 106 & 98-107 mEq/L \\
\hline Bicarbonate & 28 & $22-30 \mathrm{mEq} / \mathrm{L}$ \\
\hline Calcium & 8.2 (L) & 8.4-10.2 mg/dL \\
\hline Anion Gap & 11 & 8-12 mEq/L \\
\hline Protein, Total & 6.6 & $6.3-8.2 \mathrm{~g} / \mathrm{dL}$ \\
\hline Albumin & 4.3 & $3.5-5.0 \mathrm{~g} / \mathrm{dL}$ \\
\hline Bilirubin, Total & $1.8(H)$ & $0.2-1.3 \mathrm{mg} / \mathrm{dL}$ \\
\hline Bilirubin, Direct & $0.9(\mathrm{H})$ & $0.0-0.4 \mathrm{mg} / \mathrm{dL}$ \\
\hline Alanine Transaminase & $237(\mathrm{H})$ & 21-72 U/L \\
\hline Aspartate Transaminase & $236(H)$ & 17-59 U/L \\
\hline Alkaline Phosphatase & 124 & 38.0-126.0 U/L \\
\hline
\end{tabular}




\section{Cureus}

Lipase

\section{TABLE 1: Initial Laboratory Data}

L, Low; H, High

CT of the abdomen was performed with intravenous contrast, which demonstrated a large 10

$\mathrm{cm}$ heterogeneous hypodensity in the post right hepatic lobe with normal hepatic and portal veins and no internal gas loculations (Figure 1). It was deemed as a likely liver abscess with a mass being an unlikely possibility due to lack of biliary dilation and no mass effect.

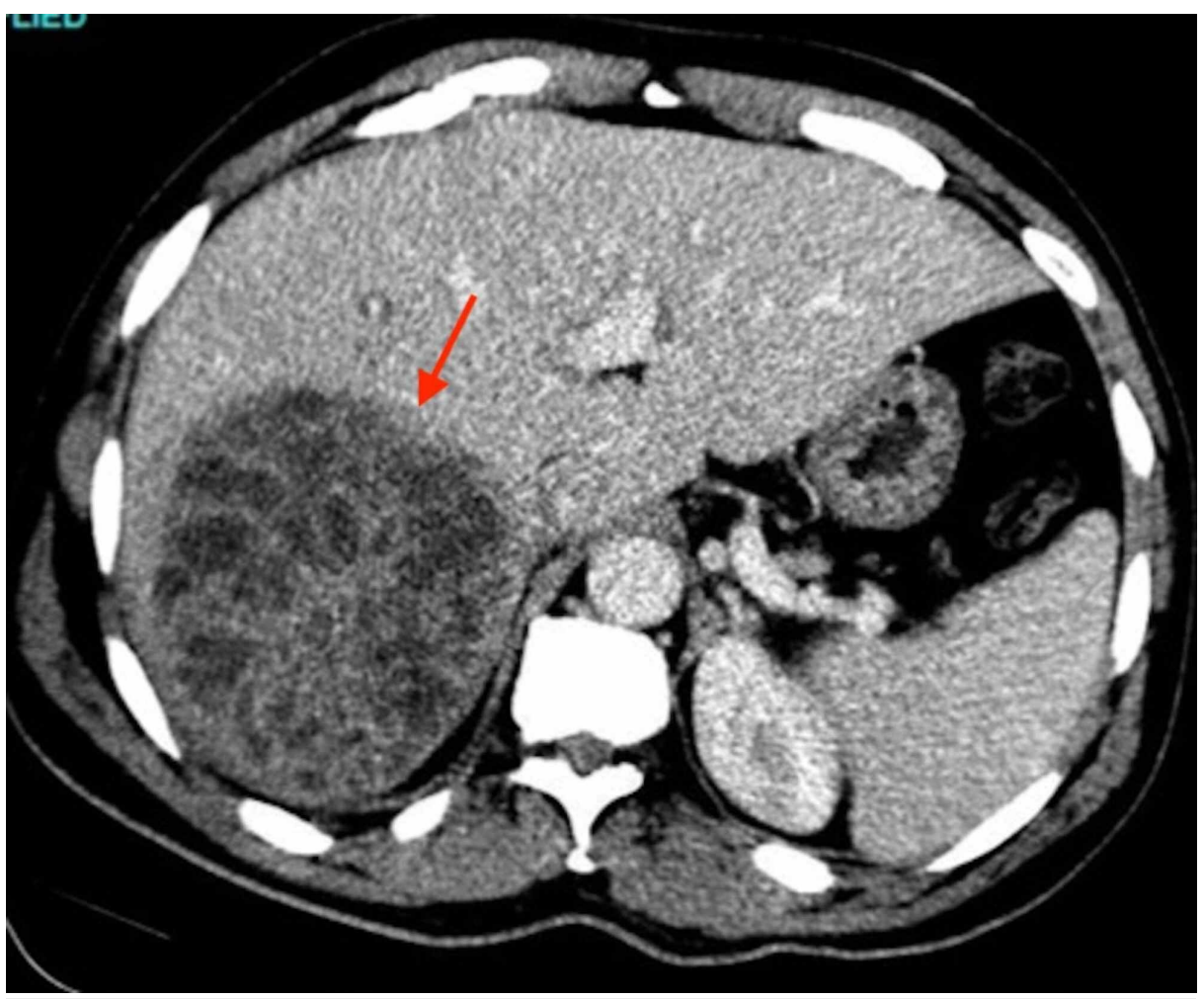

FIGURE 1: CT of the abdomen demonstrating a large $10 \mathrm{~cm}$ heterogeneous hypodensity in the post right hepatic lobe likely a liver abscess. The arrow points to area of interest.

Blood cultures drawn on the day of admission grew VGS on day 4 of admission, which was unable to grow for susceptibility testing. The patient was started on intravenous fluids for volume resuscitation and covered empirically with ceftriaxone and metronidazole. Ultrasound-guided hepatic drain placement was performed by interventional radiology, draining $100 \mathrm{~mL}$ of sanguinopurulent fluid. The aspirate fluid culture was also shown to be positive for VGS. The patient also underwent transthoracic echocardiography to rule out infectious endocarditis which did not reveal infective endocarditis. The patient was noted to have a persistently elevated international normalized ratio (INR) which peaked at 2.44 and was started on oral vitamin $\mathrm{K}$ supplementation uptitrated from $2.5 \mathrm{mg}$ daily to eventually $10 \mathrm{mg}$ which was continued daily. He later underwent ultrasonography of the abdomen on day 4 of hepatic drain placement to evaluate the abscess, which demonstrated an increase in the size of the abscess to $12.3 \times 11.9 \mathrm{~cm}$ (Figure 2). 


\section{Cureus}

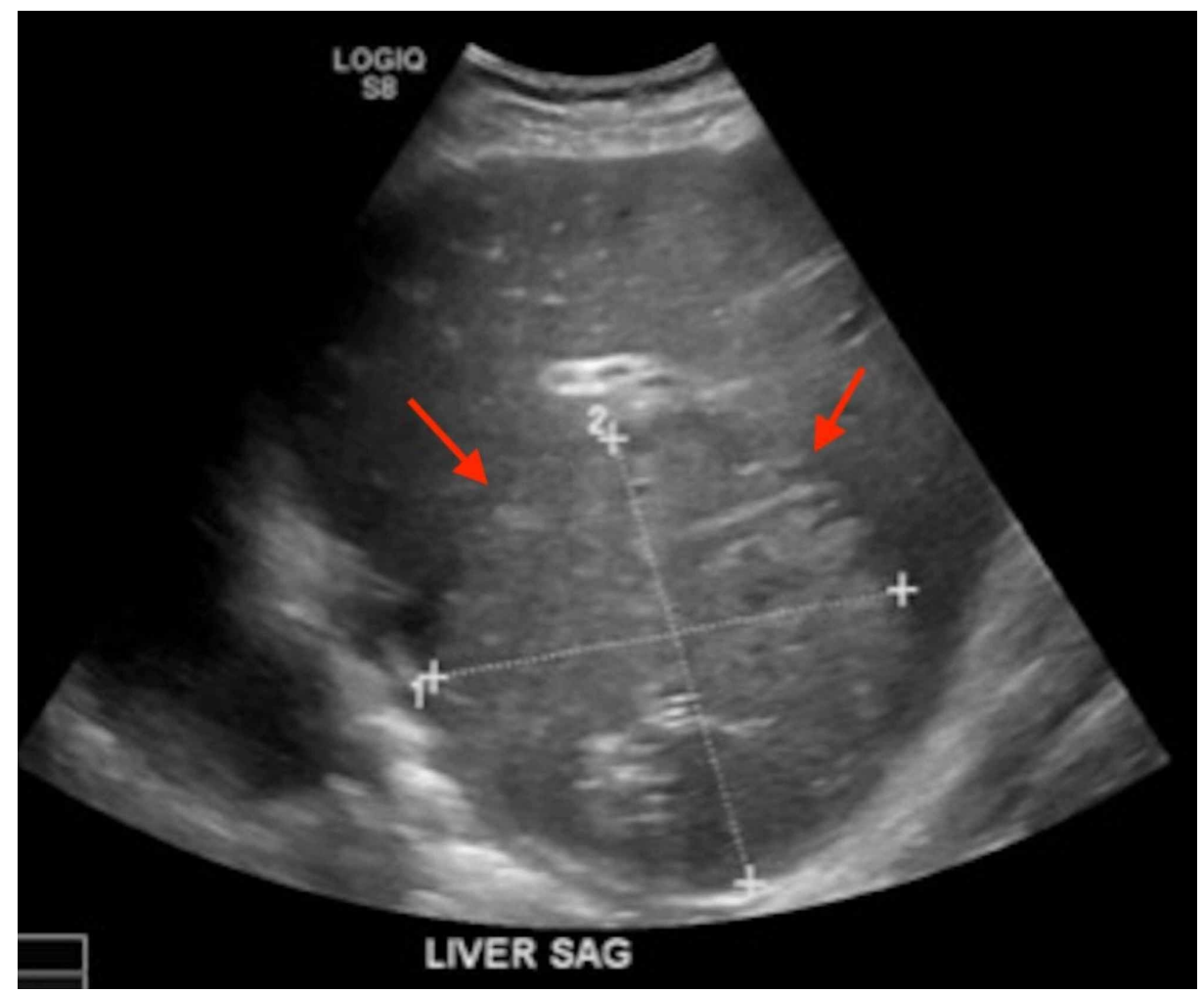

FIGURE 2: Ultrasonography of the abdomen demonstrating an increase in the size of the abscess to $12.3 \times 11.9 \mathrm{~cm}$ after hepatic drain placement. The arrows point to area of interest.

The patient subsequently underwent CT-guided placement of a large-bore pigtail catheter, and the hepatobiliary surgery department was consulted. It was recommended to obtain an MRI of the abdomen with the liver protocol, which showed a $14.0 \times 13.5 \times 1.5 \mathrm{~cm}$ multicystic enhancing mass lesion within the segments 7 and 8 of the liver with an enhancing pseudocapsule (Figure 3).

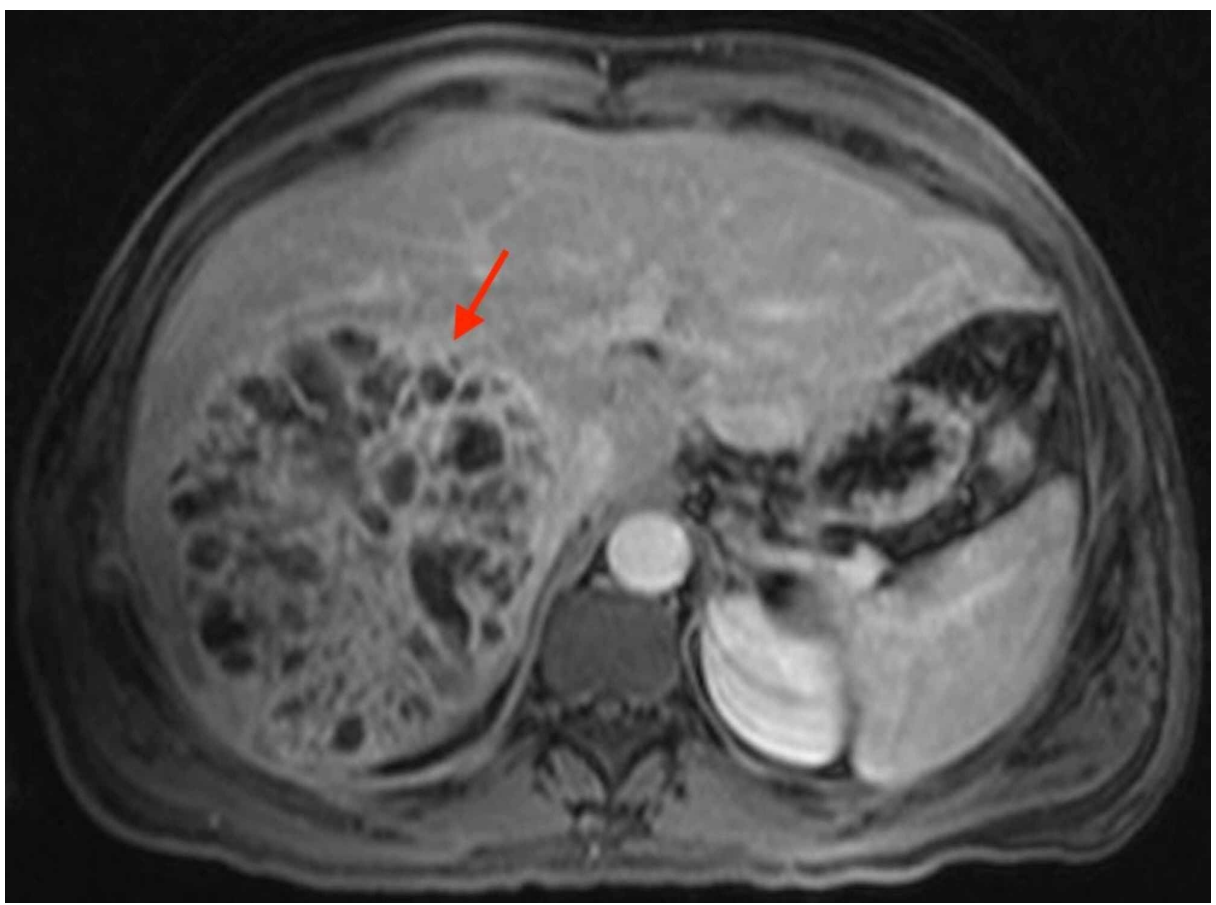

FIGURE 3: MRI of the abdomen demonstrating a $14.0 \times 13.5 \times 1.5 \mathrm{~cm}$ multicystic enhancing mass lesion within the segments 7 and 8 of the 
Numerous cystic components with enhancing walls were noted throughout the lesion. There was no infiltration within the hepatic parenchyma with non-displacement of vascular architecture. The remainder of the liver was normal in enhancement. Concerns for Echinococcus granulosus were ruled out with serological testing. Given the multiloculated nature of the abscess, failure of adequate drainage using largebore pigtail catheter, and clinical worsening of the patient with concern for sepsis, the patient was transferred to surgical service. The surgical team carried out radiology-guided drainage which drained $20 \mathrm{~mL}$ of sanguinopurulent fluid but was unsuccessful for resolution. The aspirated fluid was noted to be positive for VGS resistant to vancomycin. With the patient continuously spiking fevers and failure of improvement with radiological drainage of the abscess, surgical drainage was planned. The patient underwent right posterior liver lobectomy and appendectomy. Intraoperative findings include an abscess cavity involving segments 6, 7, and partially segment 8 of the liver with multiloculated and multiple smaller pockets (Figure $4)$.

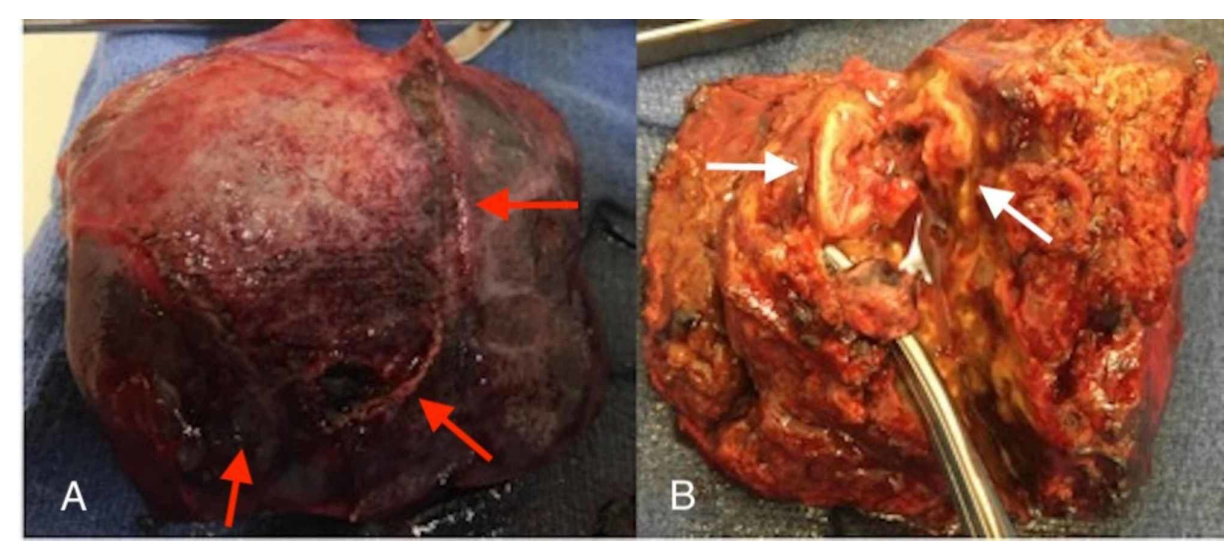

FIGURE 4: (A) Surgical specimen of right posterior liver involving segments 6,7 , and partially 8 . (B) Liver specimen demonstrating an abscess cavity with multiloculated and multiple smaller pocket. The arrows point to areas of interest.

Intraoperative specimens were sent for histopathology. Postoperatively, the patient was observed in the surgical intensive care unit. Histopathology report showed benign hepatic parenchyma with extensive acute and chronic granulomatous inflammation with no signs for malignancy. Following surgery, the patient made a quick recovery and was discharged from the hospital. He continues to follow at the outpatient clinic and is clinically doing well.

\section{Discussion}

PLAs are most often seen in developing countries and are less commonly seen in North America. The most common etiology is infection that occurs in the setting of direct extension hepatobiliary or intestinal infection and hematogenous spread [4]. Amongst the intra-abdominal causes, suppurative appendicitis is also a leading cause [5]. To our knowledge, this is the first reported case of a complicated PLA in a patient without any comorbidities which lead to hepatectomy .The most common pathogen isolated from a PLA in the United States is Escherichia coli; however, some studies indicate Klebsiella as well [6]. Members of the viridans group include Streptococcus mitis, Streptococcus mutans, Streptococcus oralis, Streptococcus sanguinis, Streptococcus sobrinus, and the Streptococcus milleri group. In one Canadian study of patients with PLA, Streptococcus millieri was the most often member of the viridans group which was identified [7]. Studies indicate an equal distribution of disease amongst males and females or a slightly higher prevalence in males as compared to females [8-10]. The pathogenic mechanism involves the failure of the initial inflammatory response to clear the insult to the liver. The classification of the abscesses is based on the route of infection which is via the hepatobiliary tree, portal vein, hepatic artery, a direct extension of infection from the contiguous area, and post-penetrating trauma to the liver [11].

Presenting symptoms include fever, chills, and abdominal pain. Laboratory abnormalities include leukocytosis ( $84 \%$ of patients), anemia (88.9\% of patients), hypoalbuminemia ( $94 \%$ of patients), and an elevated alkaline phosphatase (73\% of patients) [12]. Diagnosis is usually made by radiological imaging with ultrasonography or CT [13]. The mainstay of treatment remains antibiotics with percutaneous drainage by interventional radiology, which is effective in the majority of patients [6]. If left untreated, mortality rates of 
80\%-100\% have been reported [12]. In a retrospective study of 96 patients with PLA, it was demonstrated that in liver abscesses more than $5 \mathrm{~cm}$, surgical drainage provides better clinical outcomes than percutaneous drainage [14].

While in our case the patient was previously healthy, the majority of cases of PLA have been reported in patients in developing countries or who are immunocompromised or in patients who have underlying comorbidities such as diabetes and/or neoplasm. In our case, the patient presented with left-sided abdominal pain and weight loss. Labs were significant for leukocytosis, elevated ALT and AST >200 U/L, alkaline phosphatase, and prothrombin time/INR. The patient developed anemia and thrombocytosis, and had persistently elevated INR during the hospital course. The patient was treated with different intravenous antibiotics and underwent multiple ultrasound-guided percutaneous abscess drainages by interventional radiology, but all were unsuccessful. The patient's condition was not improving, and a repeat CT scan and MRI showed an interval increase in the size of abscess despite multiple drains placed. The surgical department was consulted as the patient was unresponsive to standard treatment modalities and was developing signs of sepsis. The patient was scheduled for right posterior lobectomy and appendectomy. Multiple units of fresh frozen plasma were transfused to correct INR prior to surgery. The patient's condition improved after surgery. Abdominal pain improved, nausea resolved, and appetite improved. The patient kept following with the hematology and surgery clinics for elevated INR as an outpatient for postoperative follow-up. On his last clinical visit, his INR is normal and he is maintaining his weight.

\section{Conclusions}

Hepatic abscesses are less common in North America. In the cases reported, most of them occur in people with preexisting medical conditions and respond to the standard treatment modality which includes a combination of antibiotics and percutaneous drainage. Our case underlines the significance of considering liver abscess as a differential even in previously healthy individuals with no known prior comorbid conditions. It also serves to indicate lobectomy as a sequela for patients who do not respond to the standard treatment modality.

\section{Additional Information \\ Disclosures}

Human subjects: Consent was obtained by all participants in this study. Conflicts of interest: In compliance with the ICMJE uniform disclosure form, all authors declare the following: Payment/services info: All authors have declared that no financial support was received from any organization for the submitted work. Financial relationships: All authors have declared that they have no financial relationships at present or within the previous three years with any organizations that might have an interest in the submitted work. Other relationships: All authors have declared that there are no other relationships or activities that could appear to have influenced the submitted work.

\section{References}

1. Erdem I, Dogru M, Emir S, et al.: Unusual presentation of Streptococcus anginosus with severe sepsis, liver abscess secondary to biliary tract perforation. J Med Cases. 2015, 6:508-511. 10.14740/jmc2326W

2. McKaigney C: Hepatic abscess: case report and review. West J Emerg Med. 2013, 14:154-157. 10.5811/westjem.2012.10.13268

3. Wu P, Chung E, Marzella N, Preis J: Streptococcus gordonii perihepatic abscess: a case report . Am J Clin Microbiol Antimicrob. 2019, 2:1038.

4. Lubbert C, Wiegand J, Karlas T: Therapy of liver abscesses. Viszeralmedizin. 2014, 30:334-341. 10.1159/000366579

5. Ochsner A, DeBakey M, Murray S: Pyogenic abscess of the liver: an analysis of forty-seven cases with review of the literature. Am J Surg. 1938, 40:292-319. 10.1016/S0002-9610(38)90618-X

6. Rahimian J, Wilson T, Oram V, Holzman RS: Pyogenic liver abscess: recent trends in etiology and mortality . Clin Infect Dis. 2004, 39:1654-1659. 10.1086/425616

7. Kaplan GG, Gregson DB, Laupland KB: Population-based study of the epidemiology of and the risk factors for pyogenic liver abscess. Clin Gastroenterol Hepatol. 2004, 2:1032-1038. 10.1016/s1542-3565(04)00459-8

8. Branum GD, Tyson GS, Branum MA, Meyers WC: Hepatic abscess. Changes in etiology, diagnosis, and management. Ann Surg. 1990, 212:655-662. 10.1097/00000658-199012000-00002

9. Huang CJ, Pitt HA, Lipsett PA, Osterman FA Jr, Lillemoe KD, Cameron JL, Zuidema GD: Pyogenic hepatic abscess: changing trends over 42 years. Ann Surg. 1996, 223:600-607. 10.1097/00000658-199605000-00016

10. Alvarez Perez JA, Gonzalez JJ, Baldonedo RF, et al.: Clinical course, treatment, and multivariate analysis of risk factors for pyogenic liver abscess. Am J Surg. 2001, 181:177-186. 10.1016/s0002-9610(00)00564-X

11. Johannsen EC, Sifri CD, Madoff LC: Pyogenic liver abscesses. Infect Dis Clin North Am. 2000, 14:547-563. 10.1016/s0891-5520(05)70120-3

12. Cellucci M, Simon E, Eppes S: Microbiology and management of pediatric liver abscesses: two cases caused by Streptococcus anginosus group. Case Rep Infect Dis. 2012, 2012:685953. 10.1155/2012/685953

13. Akuzawa N, Hatori T, Kitahara Y, Kurabayashi M: Multiple liver abscesses and bacteremia caused by Streptococcus constellatus infection: a case report. Clin Case Rep. 2017, 5:69-74. 10.1002/ccr3.774

14. Hope WW, Vrochides DV, Newcomb WL, Mayo-Smith WW, Iannitti DA: Optimal treatment of hepatic abscess. Am Surg. 2008, 74:178-182. 